\title{
Effect of five years of treatment with nipradilol eye drops in patients with normal tension glaucoma
}

This article was published in the following Dove Press journal:

Clinical Ophthalmology

25 August 201I

Number of times this article has been viewed

Kenji Inoue'

Kei Noguchi'

Masato Wakakura'

Goji Tomita²

'Inouye Eye Hospital, ${ }^{2}$ Second Department of Ophthalmology,

Toho University School of Medicine,

Tokyo, Japan
Correspondence: Kenji Inoue Inouye Eye Hospital, 4-3 Kanda-Surugadai, Chiyoda-ku, Tokyo 10I-0062, Japan

$\mathrm{Tel}+332950911$

Fax +332950917

Email inoue-k@inouye-eye.or.jp
Background: The purpose of this study was to evaluate the effects of topical nipradilol monotherapy for 5 years on intraocular pressure, visual field performance, and optic disk topography.

Methods: Thirty patients with normal tension glaucoma were monitored for intraocular pressure every 1-3 months. A Humphrey visual field test and measurement of optic disk configuration using the Heidelberg retina tomograph II was done after every year of treatment and the results compared with those before treatment. Visual field performance was also evaluated by trend and event analysis.

Results: The mean intraocular pressure \pm standard deviation after 3 years of nipradilol treatment $(14.1 \pm 2.0 \mathrm{mmHg})$ and after 5 years of nipradilol treatment $(13.7 \pm 2.1 \mathrm{mmHg})$ was significantly lower than that before treatment $(17.0 \pm 1.8 \mathrm{mmHg}, P<0.0001)$. Heidelberg retina tomograph II parameters, such as mean cup depth and height variation contour after treatment, were significantly increased compared with those before treatment. Visual field performance worsened in eight eyes by trend analysis and eight eyes by event analysis.

Conclusion: Nipradilol monotherapy was effective in reducing intraocular pressure over at least 5 years without worsening of optic disk topography. Furthermore, mean cup depth and height variation contour were also significantly improved. However, visual field performance worsened in $16.0 \%$ of patients with normal tension glaucoma.

Keywords: nipradilol, intraocular pressure, Heidelberg retina tomograph II, trend analysis, event analysis

\section{Introduction}

The only medical treatment for patients with normal tension glaucoma that shows evidence of preserving the visual fields is reduction of intraocular pressure (IOP)., ${ }^{1,2}$ Thus, IOP reduction with eye drops is generally the first-line treatment. For evaluation of the response to glaucoma therapy, IOP measurement and detection of visual field defects by visual field tests are usually conducted. Because normal tension glaucoma is a chronic progressive condition that is generally accompanied by slowly progressive visual field loss, judging the progress of this condition is difficult and long-term observation of the patient is needed. The following methods are used to evaluate the progression of visual field disorders: clinical evaluation, various evaluation criteria, ${ }^{3}$ disease stage classifications, ${ }^{4}$ linear regression (trend) analysis, ${ }^{5}$ and event analysis. ${ }^{6}$ There are various criteria for evaluating the progression of visual field loss, and results may differ depending on the criteria used. Changes in optic disk topography may precede the progression of visual field loss, but few studies have investigated 
changes in the optic disk over a long period in patients with glaucoma receiving drugs for IOP reduction and operation. ${ }^{7-10}$ Monitoring longitudinal changes of the optic disk may be useful during the treatment of glaucoma. Recent technical advances have led to the availability of methods such as scanning laser ophthalmoscopy, optical coherence tomography, or scanning laser polarimetry, which are expected to be used more in the future.

We encounter some patients who have sufficiently low IOP and who still have an IOP-lowering efficiency of 20\%-30\% from baseline, but still suffer from progressive visual field loss. Ocular circulatory dysfunction and apoptosis of retinal ganglion cells may be involved rather than IOP in those patients. Nipradilol is an antiglaucoma ophthalmic agent with nonselective $\beta$-receptor and selective $\alpha 1$-receptor blocking properties. ${ }^{11-14}$ IOP decreases because of a decline in aqueous humor production by the $\beta$ interceptor and accelerated aqueous outflow by the $\alpha$ interceptor. Nipradilol was reported to have hypotensive effects in normal tension glaucoma. ${ }^{8,15,16}$ In addition to reducing IOP, other effects of nipradilol are reported to include improvement of ocular blood flow via release of nitric oxide that causes vasodilation ${ }^{17,18}$ and neuroprotection via inhibition of apoptosis of the retinal ganglion cells. ${ }^{19,20}$ These circulatory and neuroprotective effects on the optic disk are expected to contribute to maintaining the visual fields.

The purpose of the present study was to evaluate changes in IOP, visual fields, and optic disk topography during treatment with nipradilol for normal tension glaucoma over an extended observation period of 5 years. This was an extension of our previous study of 3 years of treatment with nipradilol. ${ }^{8}$

\section{Patients and methods}

The study included 30 Japanese patients (50 eyes, 13 men and 17 women) with normal tension glaucoma who started treatment with nipradilol eye drops as monotherapy for glaucoma at Inouye Eye Hospital between April 2003 and March 2004. All patients met the enrollment criteria, did not meet any of the exclusion criteria, and were available for observation over 5 years. Their mean age was $52.4 \pm 12.5$ (range 29-76) years. Four patients had hypertension, two had diabetes, two had gout, one had hypothyroidism, and one had a sleep disorder, and all were on oral treatment for these conditions. This study was performed in accordance with the Declaration of Helsinki and was approved by our ethical committee. All patients received an explanation of the procedures and possible risks of the study, and gave their written informed consent. The criteria for diagnosing normal tension glaucoma were: IOP $\leq 21 \mathrm{mmHg}$ when measured several times to evaluate circadian variation; specific changes for glaucoma in the optic disk and the retinal nerve fiber layer (thinning of the optic disk margin and defects of the nerve fiber layer); identification of visual field defects with a high reliability and reproducibility, which were consistent with glaucomatous morphologic changes; absence of other eye diseases or congenital abnormalities that could lead to visual field changes; and normal open angle in both eyes on gonioscopy. The exclusion criteria were corrected visual acuity $\leq 0.5$, previous intraocular surgery, laser therapy, or systemic steroid therapy, otolaryngological or neurosurgical abnormalities, and diabetes mellitus. If one eye met the diagnostic criteria, the other eye was studied. If both eyes met the diagnostic criteria, then both eyes were studied. Adherence to eye drop treatment was confirmed verbally at every assessment visit. None of the patients underwent cataract surgery or any kind of intraocular surgery during the 5-year study period.

The Humphrey 30-2 SITA standard program was used for determination of the visual fields, with the mean deviation and pattern standard deviation being used as indices of visual field impairment during treatment. Patients in whom false-positive results, false-negative results, or poor fixation accounted for $20 \%$ or more of the assessments were excluded from evaluation of visual fields.

After starting treatment with nipradilol (Hypadil Kowa Ophthalmic Solution 0.25\%, Kowa, Nagoya, Japan) twice a day, IOP measurement was performed using a Goldmann applanation tonometer at the same time of day on each patient every 1-3 months. Visual field assessment (Humphrey 30-2 SITA standard program) and measurement of the optic disk were performed every 6 months. Optic nerve head topography parameters were measured using a Heidelberg retina tomograph II (HRT-II, v 3.1.2.4; Heidelberg Engineering GmbH, Heidelberg, Germany). HRT II uses a diode laser (670 nm wavelength) to scan the retinal surface sequentially in the horizontal and vertical directions on multiple focal planes. By using confocal scanning principles, a three-dimensional topographic image is acquired in consecutive focal planes. The topographic image determined from the acquired three-dimensional image consists of $384 \times 384(147,456)$ pixels, each of which is a measurement of the surface height of the retina and the optic nerve head at the corresponding location. Three topographic images were obtained, combined, and automatically aligned to create a single mean topographic 
image for analysis. Image acquisition was performed by three skilled operators, and contour lines were drawn and determined by an ophthalmologist (KI). If the standard deviation of the mean topographic image at each assessment was $>30 \mu \mathrm{m}$, the data for the patient were excluded from evaluation of morphology of the optic disk. From those results, 46 eyes were studied. The optic disk parameters included the cup/disk area ratio, rim volume $\left(\mathrm{mm}^{3}\right)$, mean cup depth $(\mathrm{mm})$, height variation contour $(\mathrm{mm})$, and mean retinal nerve fiber layer thickness $(\mathrm{mm})$.

The IOP value obtained before administration of nipradilol was compared with those obtained every 3 months after the start of treatment by repeated-measures analysis of variance and a multiple comparison test (Bonferroni/ Dunnet). The visual field parameters (mean deviation and pattern standard deviation) and HRT II parameters before administration of nipradilol were also compared with data obtained every year after the start of treatment using repeatedmeasures analysis of variance and multiple comparisons (Bonferroni/Dunnet).

\section{Statistical analysis}

The level of significance was defined as $P<0.05$ for IOP, mean deviation, analysis of variance, and HRT II parameters. Trend analysis and event analysis were applied to evaluate the progression of visual field loss. For trend analysis, linear regression was used to judge sequential changes in mean deviation, with the annual change in mean deviation $(\mathrm{dB} /$ year) being calculated along with its statistical significance. ${ }^{5}$
When test results exceeded threshold levels compared with baseline values (measured twice at the beginning of the observation period), the patient was regarded as showing progression. For event analysis, glaucoma was defined as showing "possible progression" when two consecutive measurements revealed a significant decrease by three or more points and as showing "progression" when three consecutive measurements revealed a significant decrease by three or more points. ${ }^{6}$ The time when "progression" in the present study was observed was defined as the time when the loss of visual field occurred.

\section{Results}

The mean IOP before starting treatment with nipradilol eye drops was $17.0 \pm 1.8 \mathrm{mmHg}$ (13 mmHg in one eye, $14 \mathrm{mmHg}$ in four eyes, $15 \mathrm{mmHg}$ in one eye, $16 \mathrm{mmHg}$ in 21 eyes, $18 \mathrm{mmHg}$ in 13 eyes, $19 \mathrm{mmHg}$ in four eyes, and $20 \mathrm{mmHg}$ in six eyes). The mean refractive power was $-2.1 \pm 2.8 \mathrm{D}(-8.25$ to $+2.50 \mathrm{D})$. The mean deviation at the beginning of treatment was $-6.2 \pm 6.2 \mathrm{~dB}(-25.8$ to $-1.41 \mathrm{~dB})$ and the mean pattern standard deviation was $7.7 \pm 4.5 \mathrm{~dB}(1.6$ to $16.5 \mathrm{~dB})$.

The mean IOP was $14.2 \pm 1.6,13.9 \pm 1.6,14.0 \pm 2.0$, $14.1 \pm 1.9$, and $13.7 \pm 2.1 \mathrm{mmHg}$ at years $1,2,3,4$, and 5 after starting nipradilol eye drops, respectively, and all of these values significantly decreased when compared with the pretreatment value $(P<0.0001$, Figure 1). The mean IOP reduction width was $2.8 \pm 1.7,3.1 \pm 1.7,3.0 \pm 2.0$, $2.9 \pm 1.8$, and $3.3 \pm 2.0 \mathrm{mmHg}$ at years $1,2,3,4$, and 5 after starting nipradilol eye drops, respectively, and there

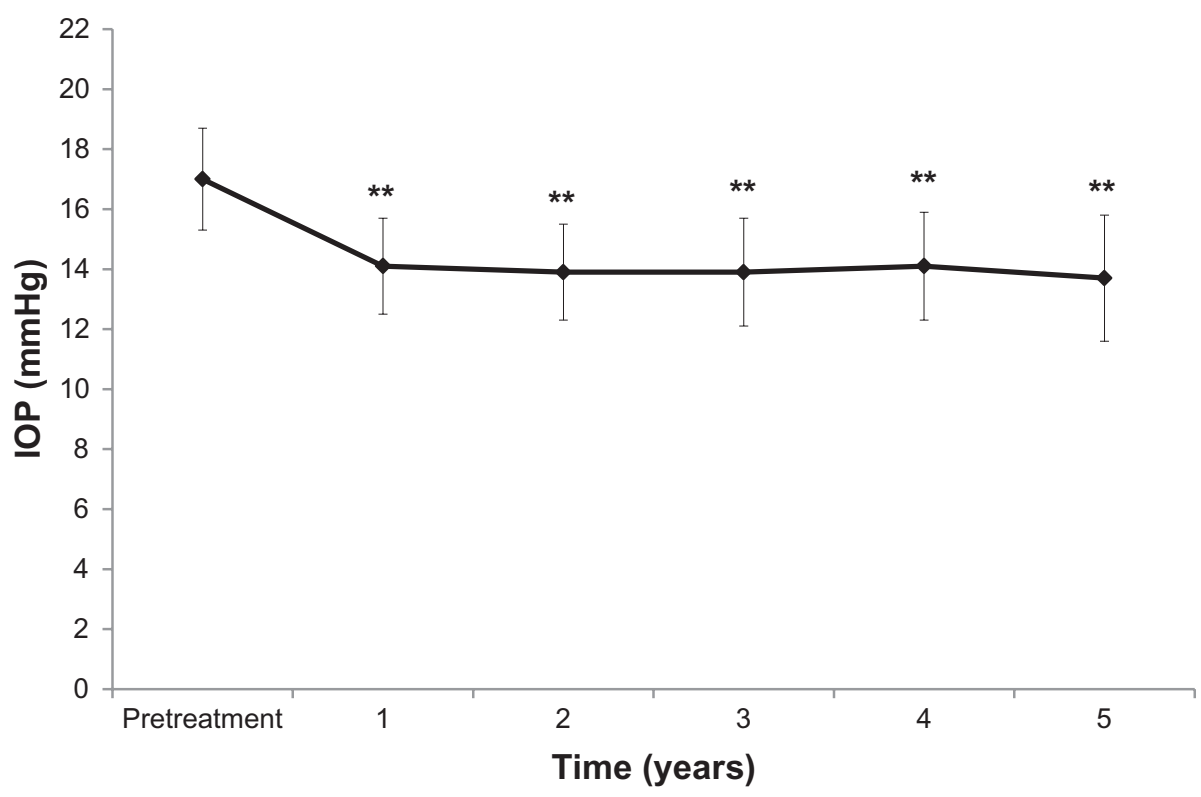

Figure I Intraocular pressure (IOP) at baseline and after treatment with nipradilol (mean \pm standard deviation). Note: ${ }^{* *} P<0.0001$, analysis of variance, Bonferroni and Dunnet multiple comparison tests. 
was a significant decrease at year 5 compared with years 1 and $4(P=0.0452)$. The mean IOP reduction rate was $16.0 \% \pm 9.3 \%, 18.1 \% \pm 8.6 \%, 17.2 \% \pm 10.3 \%, 16.7 \% \pm 9.6 \%$, and $19.0 \% \pm 10.9 \%$ at years $1,2,3,4$, and 5 after starting nipradilol eye drops, respectively, and there was a significant decrease at years 2 and 5 compared with year 1 , and at year 5 compared with years 1 and $4(P=0.0366)$.

The mean deviation values were $-6.8 \pm 6.4,-5.5 \pm 5.4$, $-5.9 \pm 5.9,-5.6 \pm 5.4$, and $-5.8 \pm 5.3 \mathrm{~dB}$ at years $1,2,3,4$, and 5 after starting nipradilol eye drops, respectively, all of which were without a significant decrease compared with the pretreatment value $(P=0.1052$, Figure 2$)$. The mean pattern standard deviation was $8.4 \pm 4.4,7.7 \pm 4.3,8.1 \pm 4.6$, $8.2 \pm 4.4$, and $8.5 \pm 4.5 \mathrm{~dB}$ at years $1,2,3,4$, and 5 after starting nipradilol eye drops, respectively, all of which were also without a significant decrease compared with the pretreatment value ( $P=0.4717$, Figure 2$)$.

Eight eyes showed "progression" in visual field loss by trend analysis, while eight eyes were evaluated by event analysis (Figure 3). Seven eyes had progression in visual field loss according to both trend analysis and event analysis. In the HRT II parameters, mean cup depth and height variation contour were significantly increased, but the other factors were not (Table 1).

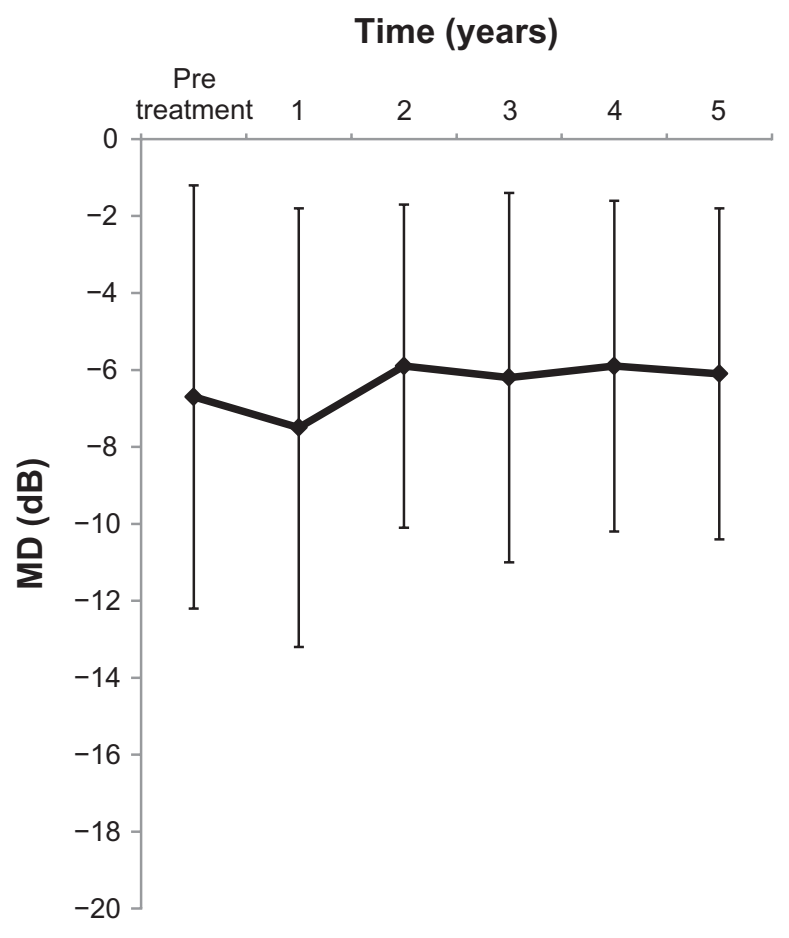

\section{Discussion}

With regards to the IOP-reducing effect of nipradilol monotherapy for normal tension glaucoma, the absolute range of IOP reduction was reported to be $0.9-3.1 \mathrm{mmHg}$ and the relative decrease of IOP was reported to be $6.4 \%-18.3 \%$. $^{8,15,16}$ Yukawa et $\mathrm{al}^{15}$ administered nipradilol for 36 months to 46 patients with a pretreatment IOP of $15.7 \pm 3.2 \mathrm{mmHg}$. They reported that the IOP significantly decreased from 13.7-14.7 $\mathrm{mmHg}$, with the range of IOP reduction being 1.0-2.0 $\mathrm{mmHg}$ and the relative decrease of IOP being $6.4 \%-12.7 \%$. Araie et $\mathrm{al}^{16}$ randomized 146 patients to receive nipradilol or timolol treatment and observed them for 3 years. In the patients treated with nipradilol, IOP showed a significant decrease from $14.1 \pm 1.7 \mathrm{mmHg}$ to $13.2 \pm 1.9 \mathrm{mmHg}$ over the 3 -year period. In our previous report, ${ }^{8}$ we determined that the IOP significantly decreased during the treatment period from $16.9 \pm 1.7 \mathrm{mmHg}$ to $13.8 \pm 14.1 \mathrm{mmHg}$, with an absolute reduction of 2.8-3.1 mmHg and a relative decrease of $16.5 \%-18.3 \%$ by administering nipradilol for 3 years to 31 patients. In the present study, the absolute range of IOP reduction was $2.9-3.3 \mathrm{mmHg}$ and the relative decrease was $17.1 \%-19.4 \%$, which were similar findings to those of our previous study ${ }^{8}$ and better compared with other reports. ${ }^{15,16}$ This difference may have been related to the higher

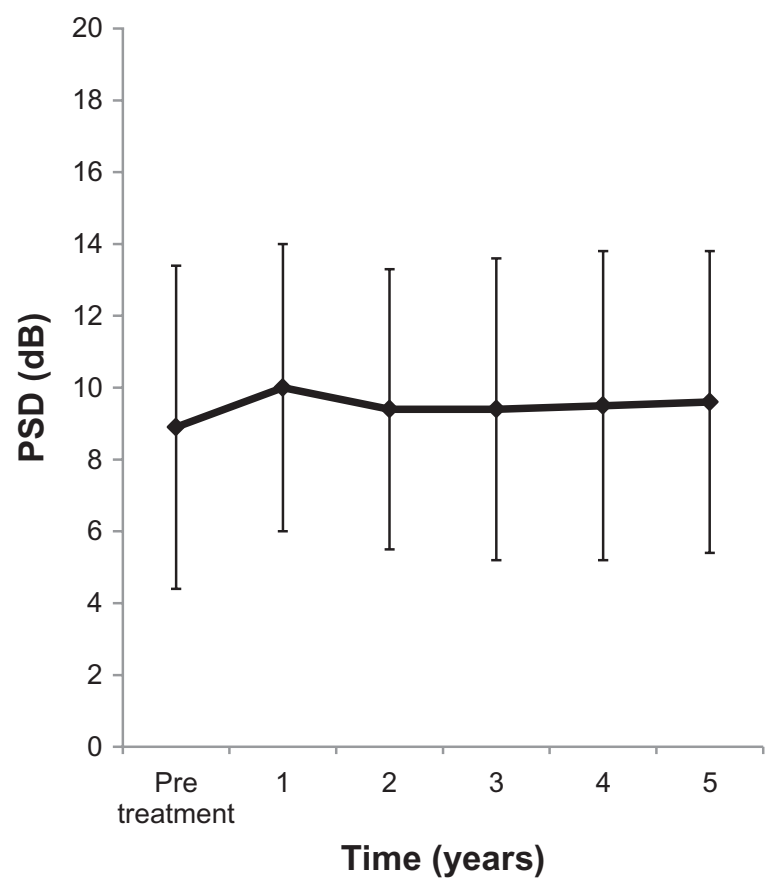

Figure 2 Mean deviation (MD) and pattern standard deviation (PSD) at baseline and years I, 2, 3, 4, and 5 of treatment with nipradilol. 


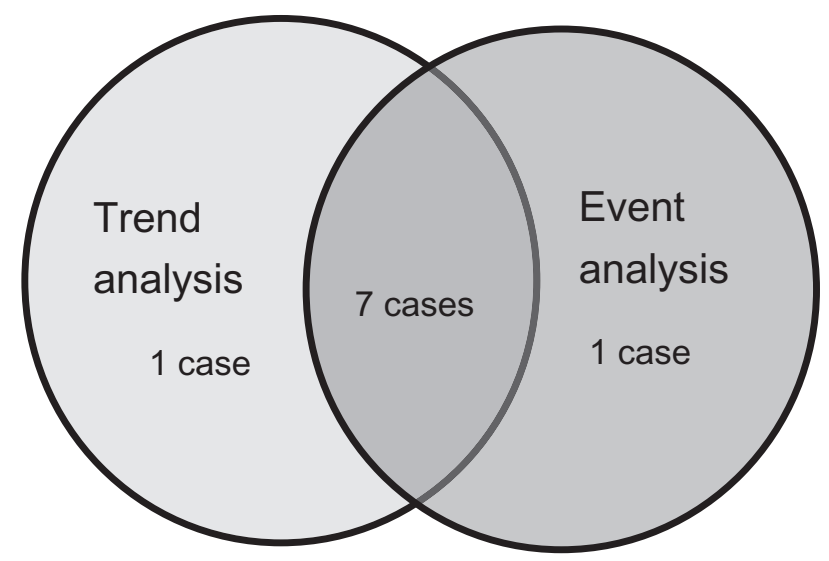

Figure 3 Analysis advanced with visual field deficit (trend analysis, event analysis).

pretreatment IOP in our present study $(17.0 \pm 1.7 \mathrm{mmHg})$ and our previous study $(16.9 \pm 1.7 \mathrm{mmHg})^{8}$ than in other studies $\left(15.7 \pm 3.2 \mathrm{mmHg}^{15}\right.$ and $\left.14.1 \pm 1.7 \mathrm{mmHg}^{16}\right)$.

Several studies have shown a visual field-preserving effect of nipradilol treatment for three or more years in patients with normal tension glaucoma. ${ }^{8,15,16}$ Yukawa et a ${ }^{15}$ reported that the visual field preservation rate was $83.2 \%$ after 4 years of treatment. They defined a decrease by $3 \mathrm{~dB}$ or more of the mean deviation calculated from two measurements obtained with Humphrey perimetry as progression of visual field loss. In the present study, the visual field preservation rate after 5 years was $86.7 \%$ when calculated by the same criterion. Araie et a ${ }^{16}$ reported that, over a 3 -year administration, no change was observed in the mean annual progression of mean deviation $(0.002 \pm 2.248 \mathrm{~dB} /$ year $)$ and a significant decrease was found in the mean annual progression of the corrected pattern standard deviation $(0.322 \pm 1.364 \mathrm{~dB} /$ year $)$. Additionally, when the visual fields were classified into six regions, the mean annual progression of total deviation was significantly improved in the two upper regions from the center, while no change was found in the other four regions (upper peripheral and lower). In the present study, evaluation by this method was not performed, but the mean annual progression of mean deviation was $-0.01 \pm 0.36 \mathrm{~dB} /$ year and there were no significant changes. The progression of visual field disorder, such as visual field preservation rate and mean annual progression of mean deviation, was no different in the present study, when compared with past reports. ${ }^{15,16}$ In the previous 3-year study of 31 patients, the mean annual progression of mean deviation was $0.09 \pm 0.83 \mathrm{~dB} /$ year, which also did not show any significant changes. ${ }^{8}$ In the present study, trend analysis and event analysis was used for assessment of visual field disorder progression. Seven of eight eyes (trend analysis) and seven of eight eyes (event analysis) both showed progression, so the results of both analyses were similar. However, no one was conscious of visual field disorder in these 50 cases.

There have been a few reports about longitudinal changes in optic disk topography in patients with glaucoma who have received IOP-reducing drugs and undergone surgery for 3 years or longer. ${ }^{7-10}$ Tomita et $\mathrm{al}^{7}$ reported patients with normal tension glaucoma who were assigned to treatment with latanoprost or timolol for 3 years. They found that the cup/disk ratio of the longitudinal diameter and the marginal area on stereographs of the optic disk showed no significant difference in both groups. Kalaboukhova et $\mathrm{al}^{9}$ reported a change in HRT parameters with glaucoma patients treated with medication and/or surgery for 50 months. Based on analyses of optic disk photographs and high-pass resolution perimetry, eyes were classified as progressive or stable. In the progressive group, HRT II parameters such as cup shape measurement, classification index, the third moment in contour, cup/disk ratio, cup area, rim area, and area below references significantly worsened. On the other hand, in the stable group, no HRT II parameters had changed significantly. Harju et $\mathrm{al}^{10}$ reported the change of HRT II parameters in glaucoma patients treated with medication and/or surgery for 6 months. Rim area and maximum cup depth had decreased significantly, and cup shape measure increased. Analysis of optic disk morphology by HRT II in the present study showed that all of the parameters at years 1, 2, 3, 4, and 5 after nipradilol treatment did not worsen compared with pretreatment values, as was shown in our previous 3-year study. ${ }^{8}$ Furthermore, the mean cup depth and height variation contour were

Table I Heidelberg retina tomograph II parameters at baseline and years I, 2, 3, 4, and 5 of treatment with nipradilol

\begin{tabular}{|c|c|c|c|c|c|c|c|}
\hline & Pretreatment & I year & 2 years & 3 years & 4 years & 5 years & $P$ \\
\hline Cup/disc area ratio & $0.50 \pm 0.18$ & $0.52 \pm 0.15$ & $0.50 \pm 0.20$ & $0.51 \pm 0.19$ & $0.52 \pm 0.17$ & $0.49 \pm 0.18$ & 0.4 \\
\hline Rim volume $\left(\mathrm{mm}^{3}\right)$ & $0.26 \pm 0.19$ & $0.29 \pm 0.18$ & $0.28 \pm 0.21$ & $0.30 \pm 0.22$ & $0.28 \pm 0.18$ & $0.31 \pm 0.19$ & 0.04 \\
\hline Mean cup depth (mm) & $0.32 \pm 0.10$ & $0.32 \pm 0.09$ & $0.31 \pm 0.10$ & $0.32 \pm 0.10$ & $0.33 \pm 0.09$ & $0.32 \pm 0.10$ & 0.12 \\
\hline Height variation contour (mm) & $0.45 \pm 0.16$ & $0.49 \pm 0.17$ & $0.50 \pm 0.18$ & $0.53 \pm 0.15$ & $0.52 \pm 0.16$ & $0.54 \pm 0.18$ & 0.01 \\
\hline Mean RNFL thickness (mm) & $0.21 \pm 0.13$ & $0.24 \pm 0.11$ & $0.23 \pm 0.14$ & $0.25 \pm 0.12$ & $0.24 \pm 0.12$ & $0.24 \pm 0.14$ & 0.05 \\
\hline
\end{tabular}

Note: $* P<0.05$, analysis of variance, Bonferroni and Dunnet multiple comparison tests. Abbreviation: RNFL, retinal nerve fiber layer. 
significantly improved. These changes in the optic nerve head are the result of nipradilol treatment. There is a hypothesis that if the morphology of the cup improves first, the rim and nerve fiber layer defect are likely to improve next. In previous reports $^{8-10}$ and in the present study, various HRT II parameters changed (worsened or improved) significantly.

Only one patient dropped out of the study between the previous 3 -year evaluation (31 patients) ${ }^{8}$ and the present 5 -year evaluation ( 30 patients). This patient discontinued treatment after 4 years because of inability to meet the study schedule. Thus, patients who have used the eye drops for 3 years or longer are unlikely to develop new adverse effects.

We assessed the effect of nipradilol for 5 years on IOP, visual field, and optic nerve head. However, because there was no control group, it is not possible to say that the changes in IOP, visual field, and optic nerve head form were attributable to nipradilol.

In conclusion, after treatment with nipradilol eye drops of 50 eyes in 30 patients with normal tension glaucoma for 5 years, there was a significant decrease of IOP, no worsened optic disk topography parameters, and progressive visual field loss in $16.0 \%$ of patients.

\section{Disclosure}

The authors report no conflicts of interest in this work.

\section{References}

1. Collaborative Normal Tension Glaucoma Study Group. Comparison of glaucomatous progression between untreated patients with normaltension glaucoma and patients with therapeutically reduced intraocular pressure. Am J Ophthalmol. 1998;126:487-497.

2. Collaborative Normal Tension Glaucoma Study Group. The effectiveness of intraocular pressure reduction in the treatment of normal-tension glaucoma. Am J Ophthalmol. 1998;126:498-505.

3. Noureddin BN, Poinoosawmy D, Fietzke FW, Hitchinqs RA. Regression analysis of visual field progression in low tension glaucoma. $\mathrm{Br} J$ Ophthalmol. 1991;75:493-495.

4. The Advanced Glaucoma Intervention Study Investigators. Advanced Glaucoma Intervention Study 2. Visual field test scoring and reliability. Ophthalmology. 1994;101:1445-1455.

5. McNaught AL, Crabb DP, Fitzke FW, Hitchinqs RA. Visual field progression: Comparison of Humphrey Statpac 2 and pointwise linear regression analysis. Graefes Arch Clin Exp Ophthalmol. 1996;234:411-418.
6. Flammer J, Drance SM, Auqustiny L, Funkhouser A. Quantification of glaucomatous visual field defects with automated perimetry. Invest Ophthalmol Vis Sci. 1985;26:176-181.

7. Tomita G, Araie M, Kitazawa Y, Tsukahara S. A three-year prospective, randomized and open comparison between latanoprost and timolol in Japanese normal-tension glaucoma patients. Eye. 2004;18:984-989.

8. Inoue K, Wakakura M, Inoue J, Tomita G. Effect of nipradilol instillation for three years in eyes with normal-tension glaucoma. Jpn J Clin Ophthalmol. 2008;62:323-327.

9. Kalaboukhova L, Fridhammar V, Lindblom B. Glaucoma follow-up by the Heidelberg retina tomograph - new graphical analysis of optic disc topography changes. Graefes Arch Clin Exp Ophthalmol. 2006;244:654-662.

10. Harju M, Saari J, Kurvinen L, Vesti E. Reversal of optic disc cupping in glaucoma. Br J Ophthalmol. 2008;92:901-905.

11. Uchida Y, Nakamura M, Shimizu S, Shirasawa Y, Fujii M. Vasoactive and beta-adrenoceptor blocking properties of 3, 4-dihydro-8(2-hydroxy-3-isopropylamino) propoxy-3-nitroxy-2H-1-benzopyran (K-351), a new antihypertensive agent. Arch Int Pharmacodyn Ther. 1983;262:132-149.

12. Ohira A, Wada Y, Fujii M, et al. Effects of nipradilol (K-351) on alphaadrenoceptor mediated responses in various isolated tissues. Arch Int Pharmacodyn Ther. 1985;278:61-71.

13. Kanno M, Araie M, Tomita K, Sawanobori K. Effects of nipradilol, a $\beta$-blocking agent with $\alpha$-blocking and nitroglycerin-like activities, on aqueous humor dynamics and fundus circulation. Invest Ophthalmol Vis Sci. 1998;39:736-743.

14. Kanno M, Araie M, Koibuchi H, Masuda K. Effects of topical nipradilol, a $\beta$ blocking agent with $\alpha$ blocking and nitroglycerin-like activities, on intraocular pressure and aqueous dynamics in humans. Br JOphthalmol. 2000;84:293-299.

15. Yukawa E, Taketani F, Matsuura T, et al. Ocular hypotensive effect of nipradilol ophthalmic solution for normal-tension glaucoma. Jpn J Clin Ophthalmol. 2003;57:1471-1475.

16. Araie M, Shirato S, Yamazaki Y, et al. Clinical efficacy of topical nipradilol and timolol on visual field performance in normal-tension glaucoma: A multicenter, randomized, double-masked comparative study. Jpn J Ophthalmol. 2008;52:255-264.

17. Taniai M, Sato E, Mizota A, Adachi-Usami E. Protective action of nipradilol against ischemia-induced retinal damage in rats. Ophthalmic Res. 2002;34:331-337.

18. Mizuno K, Koide T, Saito N, et al. Topical nipradilol: Effects on optic nerve head circulation in humans and periocular distribution in monkeys. Invest Ophthalmol Vis Sci. 2002;43:3243-3250.

19. Kashiwagi K, Lizuka Y, Tsukahara S. Neuroprotective effects of nipradilol on purified cultured retinal ganglion cells. J Glaucoma. 2002;11:231-238.

20. Mizuno K, Koide T, Yoshimura M, Araie M. Neuroprotective effect and intraocular penetration of nipradilol, a beta-blocker with nitric oxide donative action. Invest Ophthalmol Vis Sci. 2001;42:688-694.
Clinical Ophthalmology

\section{Publish your work in this journal}

Clinical Ophthalmology is an international, peer-reviewed journal covering all subspecialties within ophthalmology. Key topics include: Optometry; Visual science; Pharmacology and drug therapy in eye diseases; Basic Sciences; Primary and Secondary eye care; Patient Safety and Quality of Care Improvements. This journal is indexed on Submit your manuscript here: http://www.dovepress.com/clinical-ophthalmology-journal

\section{Dovepress}

PubMed Central and CAS, and is the official journal of The Society of Clinical Ophthalmology (SCO). The manuscript management system is completely online and includes a very quick and fair peer-review system, which is all easy to use. Visit http://www.dovepress.com/ testimonials.php to read real quotes from published authors. 